\title{
Beyond Set Theory in Bell Inequality
}

\author{
Germano Resconi ${ }^{1}$, Koji Nagata ${ }^{2}$ \\ ${ }^{1}$ Department of Mathematics and Physics, Catholic University, Brescia, Italy \\ ${ }^{2}$ Department of Physics, Korea Advanced Institute of Science and Technology, Daejeon, Korea \\ Email: resconi@speedyposta.it, ko_mi_na@yahoo.co.jp
}

Received 10 December 2015; accepted 15 January 2016; published 19 January 2016

Copyright (C) 2016 by authors and Scientific Research Publishing Inc.

This work is licensed under the Creative Commons Attribution International License (CC BY).

http://creativecommons.org/licenses/by/4.0/

(c) (i) Open Access

\section{Abstract}

Feynman pointed out a logic and mathematical paradox in particle physics. The paradox is that we get for the same entity only local dependence and global dependence at the time. This contradiction is coming from the dual nature of the particle viewed as a wave. In the first capacity it has only local dependence; in the second (wave) capacity it has a global dependence. The classical logic has difficulties in resolving this paradox. Changing the classical logic to logic makes the paradox apparent. Particle has the local property or zero dependence with other particles, media has total dependence so it is a global unique entity. Now, in set theory, any element is independent from the other so disjoint set has no elements in common. With this condition we have known that the true/ false logic can be applied and set theory is the principal foundation. Now with conditional probability and dependence by copula the long distance dependence has an effect on any individual entity that now is not isolate but can have different types of dependence or synchronism (constrain) whose effect is to change the probability of any particle. So particle with different degree of dependence can be represented by a new type of set as fuzzy set in which the boundary is not completely defined or where we cannot separate a set in its parts as in the evidence theory. In conclusion the Feynman paradox and Bell violation can be explained at a new level of complexity by many valued logics and new types of set theory.

\section{Keywords}

Quantum Non Locality, Quantum Entanglement, Formalism

\section{Bell Inequality}

Let $X=\left\{x_{1}, x_{2}, \cdots, x_{n}\right\}$ be a set of elements with joint probabilities

$$
p_{j}\left(x_{j}\right), p_{i, j}\left(x_{i}, x_{j}\right), p_{i, j, k}\left(x_{i}, x_{j}, x_{k}\right), \cdots
$$


For example, consider $X=\left\{x_{1}, x_{2}, x_{3}\right\}$ and a power set

$$
2^{X}=\left\{\varnothing,\left\{x_{1}\right\},\left\{x_{2}\right\},\left\{x_{3}\right\},\left\{x_{1}, x_{2}\right\},\left\{x_{1}, x_{3}\right\},\left\{x_{2}, x_{3}\right\},\left\{x_{1}, x_{2}, x_{3}\right\}\right\}
$$

In the classical probability calculus this power set can be rewritten as

$$
\begin{gathered}
2^{X}=\left\{\varnothing,\left\{x_{1}\right\},\left\{x_{2}\right\},\left\{x_{3}\right\},\left\{x_{1}, x_{2}\right\}=\left\{x_{1}\right\} \cup\left\{x_{2}\right\},\left\{x_{1}, x_{3}\right\}=\left\{x_{1}\right\} \cup\left\{x_{3}\right\},\right. \\
\left.\left\{x_{2}, x_{3}\right\}=\left\{x_{2}\right\} \cup\left\{x_{3}\right\},\left\{x_{1}, x_{2}, x_{3}\right\}=\left\{x_{1}\right\} \cup\left\{x_{2}\right\} \cup\left\{x_{3}\right\}\right\}
\end{gathered}
$$

Also in the classical probability theory $p(A \cup B)=p(A)+p(B)-p(A \cap B)$ and the probability of a set is the sum of probabilities of its elements:

$$
\begin{aligned}
& \left\{p(\varnothing)=0, p\left(\left\{x_{1}\right\}\right), p\left(\left\{x_{2}\right\}\right), p\left(\left\{x_{3}\right\}\right), p\left(\left\{x_{1}, x_{2}\right\}\right)=p\left(\left\{x_{1}\right\}\right)+p\left(\left\{x_{2}\right\}\right), p\left(\left\{x_{1}, x_{3}\right\}\right)=p\left(\left\{x_{1}\right\}\right)+p\left(\left\{x_{3}\right\}\right),\right. \\
& \left.p\left(\left\{x_{2}, x_{3}\right\}\right)=p\left(\left\{x_{2}\right\}\right)+p\left(\left\{x_{3}\right\}\right), p\left(\left\{x_{1}, x_{2}, x_{3}\right\}\right)=p\left(\left\{x_{1}\right\}\right)+p\left(\left\{x_{2}\right\}\right)+p\left(\left\{x_{3}\right\}\right)\right\}
\end{aligned}
$$

because the intersection of elementary events is empty.

$$
S=\left[\begin{array}{c}
A \cap B \cap C \\
A^{C} \cap B \cap C \\
A \cap B^{C} \cap C \\
A \cap B \cap C^{C} \\
A^{C} \cap B^{C} \cap C \\
A^{C} \cap B \cap C^{C} \\
A \cap B^{C} \cap C^{C} \\
A^{C} \cap B^{C} \cap C^{C}
\end{array}\right]
$$

In a graphic way it is shown in Figure 1.

\section{These sets have the following Bell inequality.}

$$
\begin{aligned}
& \left(A \cap B^{C}\right) \cup\left(B \cap C^{C}\right)=S_{1} \cup S_{7} \cup S_{4} \cup S_{6} \\
& A \cap C^{C}=S_{7} \cup S_{4} \\
& A \cap C^{C} \subseteq\left(A \cap B^{C}\right) \cup\left(B \cap C^{C}\right) \\
& \text { and }\left|A \cap C^{C}\right| \leq\left|\left(A \cap B^{C}\right) \cup\left(B \cap C^{C}\right)\right|
\end{aligned}
$$

Now we introduce dependence between events. Consider an event with property $A$ and another event with a negated property $A^{C}$. These events can be called dependent (correlated). This dependence takes place for particles.

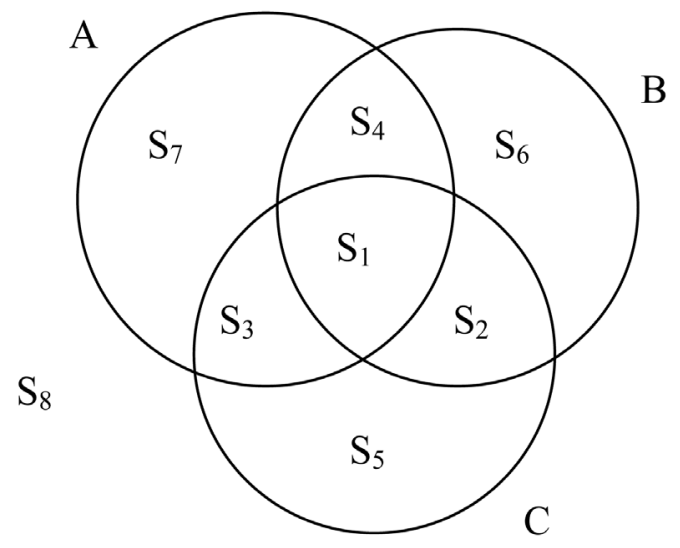

Figure 1. Set theory intersections or elements. 
Consider an event with property $A \cap C^{C}$ that is with both properties $A$ and $C^{C}$ at the same time. We cannot measure the two properties by using one instrument at the same time, but we can use the correlation to measure the second property if two properties are correlated. We can also view an event with property $A \cap C^{C}$ as two events: event $e_{A}$ with property $A$ and $e_{C^{C}}$ with the property $C$ in the opposite state (negated). The number of pairs of events $\left(e_{A}, e_{C^{C}}\right)$ is the same as the number of events with the superposition of $A$ and $C^{C}$, $A \cap C^{C}$. In this condition d'Espagnat explains the connection between the set theory and Bell's inequality. It is known that the Bell's inequality that gives us the reality condition is violated. Conclusion: The Bell inequality is based on the classical set theory that is connected with the classical logic. The set theory assumes empty overlap (as a form of independence) of elementary elements which is the basis for the Bell inequality. Thus the logic of dependence can differ from the logic of independence. Thus we must use a theory beyond the classical set theory.

\section{Dependence and Independence in the Double Slit Experiment as Physical Image of Copula and Fuzzy}

The goal of this section is to analyze the double slit experiment [1] as a demonstration of the need to build a separate theory to deal with dependent/related evens under uncertainty. The design and results of the double slits experiment is outlined in Figure 2(A) and Figure 2(B) [2], where points in Figure 2(B) show particles (elementary probability event) that pass slits.

Figure 2(C) shows theoretical result of the double slit expreiment when only the set theory is used to combine events: one event $e_{1}$ for one slid and another event $e_{2}$ for the second slid. In this set-theoretical approach it is assumed that events $e_{1}$ and $e_{2}$ are elementary events that do not overlap (have empty intersection, "incompatible", completely independent). In this case, the probability that either one of these two events will occur is

$$
p\left(e_{1} \cup e_{2}\right)=p\left(e_{1}\right)+p\left(e_{2}\right)
$$

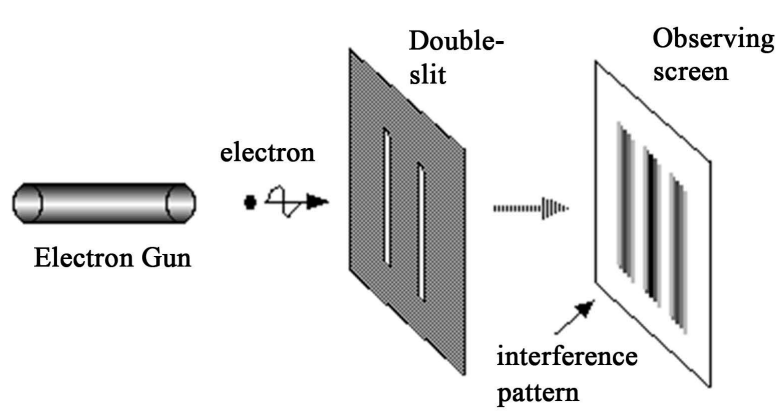

(A)

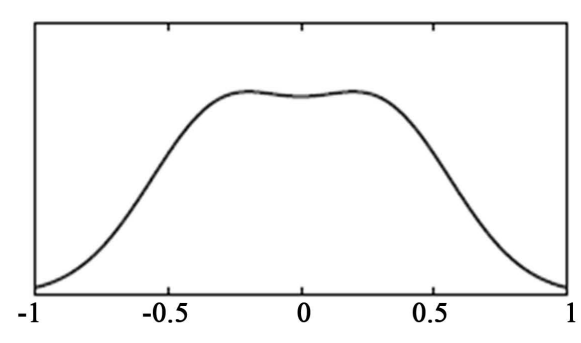

(C)

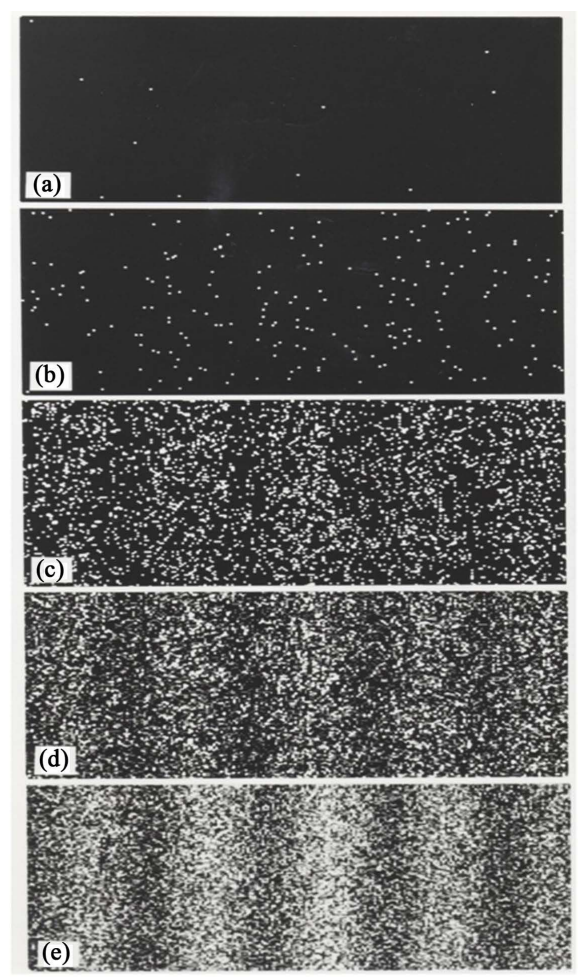

(B)

Figure 2. (A) Double slit experiment design; (B) Result of double slit experiment: electron buildup over time; (C) Distribution of independent particles (events). 
In classical logic it is always true that variable is self-dependent (that is the repeat of the process produces the same result). In the probability calculus it is not the case. The random factors can change the output when the situation is repeated. Quite often the probabilistic approach is applied to study frequency of independent phenomena. In the case of dependent variables we cannot derive $p\left(x_{1}, x_{2}\right)$ as a product of independent probabilities, $p\left(x_{1}\right) p\left(x_{2}\right)$ and must use multidimensional probability distribution with dependent valuables. The common technique for modeling it is a Bayesian network. In the Bayesian approach the evidence about the true state of the world is expressed in terms of degrees of belief in the form of Bayesian conditional probabilities. The conditional probability is the main element to express the dependence or inseparability of the two states $x_{1}$ and $x_{2}$ in the probability theory. The joint probability $p\left(x_{1}, x_{2}, \cdots, x_{n}\right)$ is represented via multiple conditional probabilities to express the dependence between variables. The copula approach introduces a single function $c\left(u_{1}, u_{2}\right)$ denoted as density of copula as a way to model the dependence or inseparability of the variables with the following property in the case of two variables. The copula allows representing the joint probability $p\left(x_{1}, x_{2}\right)$ as a combination (product) of single dependent part $c\left(u_{1}, u_{2}\right)$ and independent parts: probabilities $p\left(x_{1}\right)$ and $p\left(x_{2}\right)$. The investigation of copulas and their applications is a rather recent subject of mathematics. From one point of view, copulas are functions that join or "couple" one-dimensional distribution functions $u_{1}$ and $u_{2}$ and the corresponding joint distribution function.

\section{Conditional Probability, Dependence in Probability Calculus and Copula}

\section{A joint probability distribution}

$$
p\left(x_{1}, x_{2}, \cdots, x_{n}\right)=p\left(x_{1}\right) p\left(x_{2} \mid x_{1}\right) p\left(x_{3} \mid x_{2}, x_{1}\right) \cdots p\left(x_{n} \mid x_{1}, x_{2}, \cdots, x_{n-1}\right),
$$

e.g., for two variables $p\left(x_{1}, x_{2}\right)=p\left(x_{1}\right) p\left(x_{2} \mid x_{1}\right)$. A function $c\left(u_{1}, u_{2}\right)$ is a density of copula

$$
\text { if } p\left(x_{1}, x_{2}\right)=c\left(u_{1}, u_{2}\right) p\left(x_{1}\right) p\left(x_{2}\right)=p\left(x_{1}\right) p\left(x_{2} \mid x_{1}\right)
$$

where $u_{1}=\int p\left(x_{1}\right) \mathrm{d} x_{1}$ and $u_{2}=\int p\left(x_{2}\right) \mathrm{d} x_{2}$.

A cumulative function $C$ with inverse functions $x_{i}\left(u_{i}\right)$ as arguments:

$$
\begin{aligned}
C\left(x_{1}\left(u_{1}\right), x_{2}\left(u_{2}\right)\right) & =\int p\left(x_{1}, x_{2}\right) \mathrm{d} x_{1} \mathrm{~d} x_{2}=\int c\left[u_{1}\left(x_{1}\right), u_{2}\left(x_{2}\right)\right] p\left(x_{1}\right) p\left(x_{2}\right) \mathrm{d} x_{1} \mathrm{~d} x_{2} \\
& =\int p\left(x_{1}\right) p\left(x_{2} \mid x_{1}\right) \mathrm{d} x_{1} \mathrm{~d} x_{2}
\end{aligned}
$$

where $p\left(x_{1}\right)=\frac{\mathrm{d} u_{1}}{\mathrm{~d} x_{1}}, p\left(x_{2}\right)=\frac{\mathrm{d} u_{2}}{\mathrm{~d} x_{2}}$ and respectively inverse functions $u_{1}\left(x_{1}\right)=\int p\left(x_{1}\right) \mathrm{d} x_{1}, u_{2}\left(x_{2}\right)=\int p\left(x_{2}\right) \mathrm{d} x_{2}$

An alternative representation of a cumulative function $C$

$$
\begin{aligned}
& C\left(x_{1}\left(u_{1}\right), x_{2}\left(u_{2}\right)\right)=C\left(u_{1}, u_{2}\right)=\int c\left(u_{1}, u_{2}\right) p\left(x_{1}\right) \mathrm{d} x_{1} p\left(x_{2}\right) \mathrm{d} x_{2}=\int c\left(u_{1}, u_{2}\right) \mathrm{d} u_{1} \mathrm{~d} u_{2} \\
& \text { and } c\left(u_{1}, u_{2}\right)=\frac{\partial^{2} C\left(u_{1}, u_{2}\right)}{\partial u_{1} \partial u_{2}}
\end{aligned}
$$

\section{Copula properties.}

2-D case

$$
p\left(x_{1}\right) p\left(x_{2} \mid x_{1}\right)=\frac{\partial^{2} C\left(u_{1}, u_{2}\right)}{\partial u_{1} \partial u_{2}} p\left(x_{1}\right) p\left(x_{2}\right), p\left(x_{2} \mid x_{1}\right)=\frac{\partial^{2} C\left(u_{1}, u_{2}\right)}{\partial u_{1} \partial u_{2}} p\left(x_{2}\right)
$$

3-D case

$$
\begin{aligned}
p\left(x_{1}, x_{2}, x_{n}\right) & =p\left(x_{1}\right) p\left(x_{2} \mid x_{1}\right) p\left(x_{3} \mid x_{2}, x_{1}\right)=c\left(u_{1}, u_{2}, u_{3}\right) p\left(x_{1}\right) p\left(x_{2}\right) p\left(x_{3}\right) \\
C\left(u_{1}, u_{2}, u_{3}\right) & =\int p\left(x_{1}, x_{2}, x_{3}\right) \mathrm{d} x_{1} \mathrm{~d} x_{2} \mathrm{~d} x_{3}=\int c\left(u_{1}, u_{2}, u_{3}\right) p\left(x_{1}\right) p\left(x_{2}\right) p\left(x_{3}\right) \mathrm{d} x_{1} \mathrm{~d} x_{2} \mathrm{~d} x_{3} \\
& =\int p\left(x_{1}\right) p\left(x_{2} \mid x_{3}\right) p\left(x_{3} \mid x_{2}, x_{3}\right) \mathrm{d} x_{1} \mathrm{~d} x_{2} \mathrm{~d} x_{3}
\end{aligned}
$$

for $p\left(x_{1}\right)=\frac{\mathrm{d} u_{1}}{\mathrm{~d} x_{1}}, p\left(x_{2}\right)=\frac{\mathrm{d} u_{2}}{\mathrm{~d} x_{2}}, p\left(x_{3}\right)=\frac{\mathrm{d} u_{3}}{\mathrm{~d} x_{3}}$ 


$$
\begin{aligned}
& C\left(u_{1}, u_{2}, u_{3}\right)=\int c\left(u_{1}, u_{2}, u_{3}\right) p\left(x_{1}\right) \mathrm{d} x_{1} p\left(x_{2}\right) \mathrm{d} x_{2} p\left(x_{3}\right) \mathrm{d} x_{3}=\int c\left(u_{1}, u_{2}, u_{3}\right) \mathrm{d} u_{1} \mathrm{~d} u_{2} \mathrm{~d} u_{3} \\
& \text { so } c\left(u_{1}, u_{2}, u_{3}\right)=\frac{\partial^{3} C\left(u_{1}, u_{2}, u_{3}\right)}{\partial u_{1} \partial u_{2} \partial u_{3}} \\
& p\left(x_{1}, x_{2}, x_{n}\right)=p\left(x_{1}\right) p\left(x_{2} \mid x_{1}\right) p\left(x_{3} \mid x_{2}, x_{1}\right)=\frac{\partial^{3} C\left(u_{1}, u_{2}, u_{3}\right)}{\partial u_{1} \partial u_{2} \partial u_{3}} p\left(x_{1}\right) p\left(x_{2}\right) p\left(x_{3}\right) \\
& \text { and } p\left(x_{2} \mid x_{1}\right) p\left(x_{3} \mid x_{2}, x_{1}\right)=\frac{\partial^{2} C\left(u_{1}, u_{2}, u_{3}\right)}{\partial u_{1} \partial u_{2}} p\left(x_{2}\right) p\left(x_{3} \mid x_{2}, x_{1}\right)=\frac{\partial^{3} C\left(u_{1}, u_{2}, u_{3}\right)}{\partial u_{1} \partial u_{2} \partial u_{3}} p\left(x_{2}\right) p\left(x_{3}\right) \\
& \text { and } \frac{\partial^{2} C\left(u_{1}, u_{2}, u_{3}\right)}{\partial u_{1} \partial u_{2}} p\left(x_{3} \mid x_{2}, x_{1}\right)=\frac{\partial^{3} C\left(u_{1}, u_{2}, u_{3}\right)}{\partial u_{1} \partial u_{2} \partial u_{3}} p\left(x_{3}\right) \\
& \text { and } p\left(x_{3} \mid x_{2}, x_{1}\right)=\frac{\partial^{3} C\left(u_{1}, u_{2}, u_{3}\right)}{\partial u_{1} \partial u_{2} \partial u_{3}} \frac{1}{\frac{\partial^{2} C\left(u_{1}, u_{2}, u_{3}\right)}{\partial u_{1} \partial u_{2}}} p\left(x_{3}\right)
\end{aligned}
$$

\section{General n-D case}

$$
p\left(x_{n} \mid x_{1}, x_{2}, \cdots, x_{n-1}\right)=\frac{\partial^{n} C\left(u_{1}, u_{2}, \cdots, u_{n}\right)}{\partial u_{1}, \cdots, \partial u_{n}} \frac{1}{\frac{\partial^{n-1} C\left(u_{1}, u_{2}, \cdots, u_{n}\right)}{\partial u_{1}, \cdots, \partial u_{n-1}}} p\left(x_{n}\right)
$$

\section{Conditional copula:}

$$
c\left(x_{n} \mid x_{1}, x_{2}, \cdots, x_{n-1}\right)=\frac{\partial^{n} C\left(u_{1}, u_{2}, \cdots, u_{n}\right)}{\partial u_{1}, \cdots, \partial u_{n}} \frac{1}{\frac{\partial^{n-1} C\left(u_{1}, u_{2}, \cdots, u_{n}\right)}{\partial u_{1}, \cdots, \partial u_{n-1}}}
$$

where $\frac{\partial^{n} C\left(u_{1}, u_{2}, \cdots, u_{n}\right)}{\partial u_{1}, \cdots, \partial u_{n}}=c\left(u_{1}, u_{2}, \cdots, u_{n}\right)$

In literature commonly $C\left(u_{1}, u_{2}, \cdots, u_{n}\right)$ is denoted as copula and $c\left(u_{1}, u_{2}, \cdots, u_{n}\right)$ is denoted as a density of copula.

\section{Examples of Copula and Dependence}

When $u(x)$ is a marginal probability $F(x), u(x)=F(x)$ and $u$ is uniformly distributed then the inverse function $x(u)$ is not uniformly distributed, but has values concentrated in the central part as the Gaussian distribution. The inverse process is represented graphically in Figures 3-5.

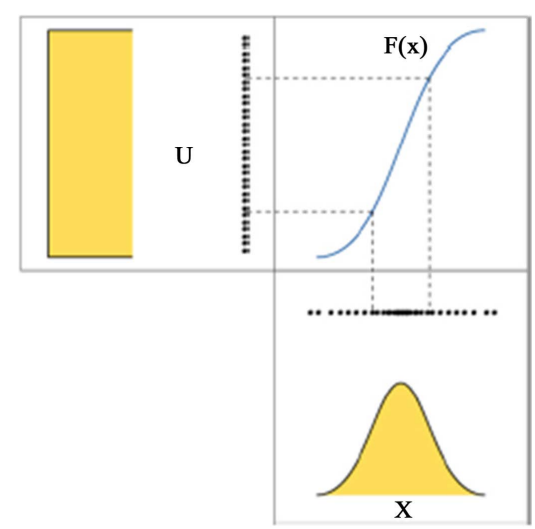

Figure 3. Relation between marginal probability $F(x)$ and the random variable $x$. 


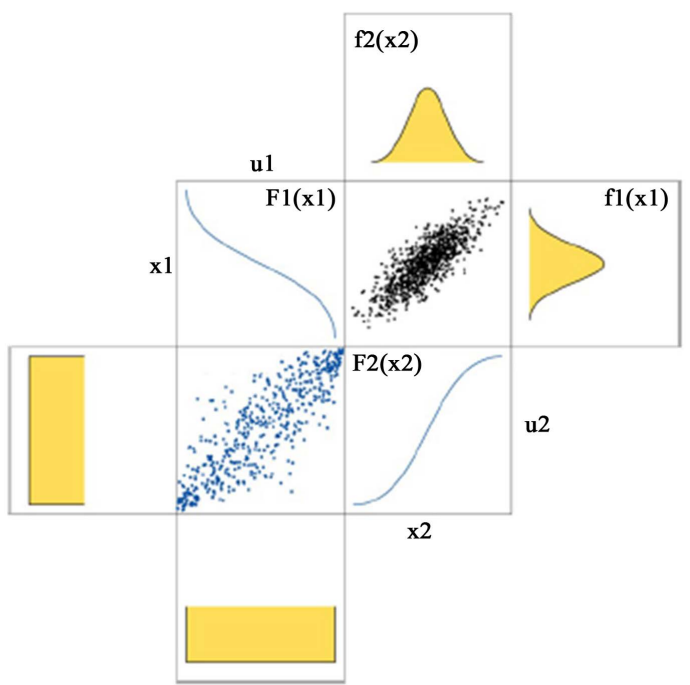

Figure 4. Symmetric joint probability and copula.

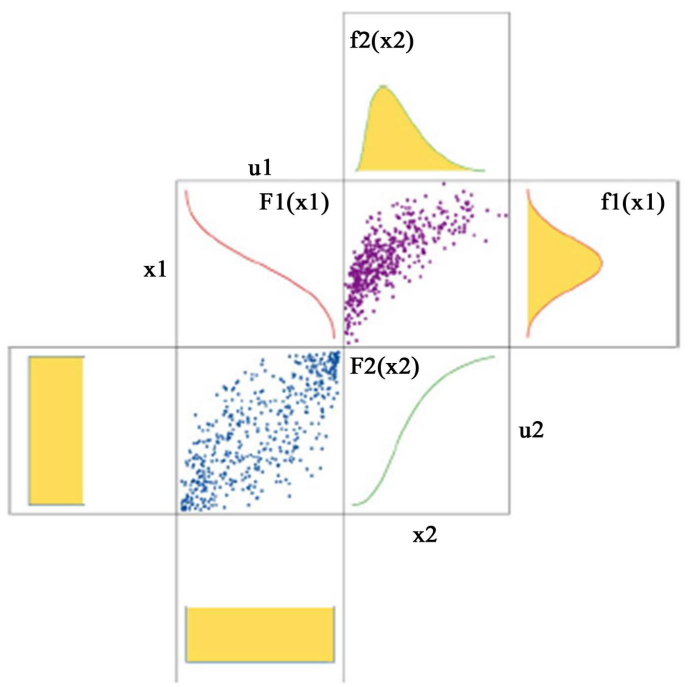

Figure 5. Asymmetric joint probability and symmetric copula.

Consider another example where a joint probability density function $p$ is defined in the two dimensional interval $(0,2) \times(0,1)$ as follows,

$$
p(x, y)=\frac{x+y}{3}
$$

Then the marginal function in this interval is

$$
C(x, y)=\int p(x, y) \mathrm{d} x \mathrm{~d} y=\int \frac{x+y}{3} \mathrm{~d} x \mathrm{~d} y=\frac{x y(x+y)}{6}
$$

Next we change the reference

$$
p\left(x_{1}\right)=\frac{\mathrm{d} u_{1}}{\mathrm{~d} x_{1}}, p\left(x_{2}\right)=\frac{\mathrm{d} u_{2}}{\mathrm{~d} x_{2}}
$$

and use the marginal probabilities

$$
u_{1}=\int p(x) \mathrm{d} x, u_{2}=\int p(y) \mathrm{d} y
$$


to get

$$
\begin{gathered}
u_{1}(x)=C(x, 1)=\frac{x(x+1)}{6}, x \in[0,2] \\
u_{2}=C(2, y)=\frac{y(y+2)}{6}, y \in[0,1]
\end{gathered}
$$

This allows us computing the inverse function to identify variables $x$ and $y$ as functions of the marginal functions $u_{1}$ and $u_{2}$ :

$$
\begin{aligned}
& x\left(u_{1}\right)=\frac{\sqrt{1+24 u_{1}}-1}{2} \\
& y\left(u_{2}\right)=\sqrt{1+3 u_{2}}-1
\end{aligned}
$$

Then these values are used to compute the copula $C$ in function (1)

$$
\begin{aligned}
C\left(u_{1}, u_{2}\right) & =\frac{\sqrt{1+24 u_{1}}-1}{2}\left(\sqrt{1+3 u_{2}}-1\right) \frac{\frac{\sqrt{1+24 u_{1}}-1}{2}+\left(\sqrt{1+3 u_{2}}-1\right)}{6} \\
& =\frac{\sqrt{1+24 u_{1}}-1}{2}\left(\sqrt{1+3 u_{2}}-1\right) \frac{\frac{\sqrt{1+24 u_{1}}-1}{2}+\left(\sqrt{1+3 u_{2}}-1\right)}{6}
\end{aligned}
$$

\section{Physical Paradox and Physical Meaning of Copula and Fuzzy Theory}

Feynman's argument [3] involves the idea that classically we think in terms of two distinct and incompatible concepts, particles and waves. These concepts are incompatible because particles are localized and waves are not. To see this, let us start with a point particle or elementary event. In classical mechanics, particles are objects localized in space, and therefore, can only interact with systems that local for them. If a particle then collides with another particle, say constituent of a wall placed in the way of the original particle, an interaction will occur. However, as soon as the particle loses contact with the wall, the interaction ceases to exist. In other words, particles interact locally or have local not global dependence. The second basic concept is the concept of waves. Historically, the physics describing a point particle was extended to include the description of continuous media, and, more importantly to our current discussion, the vibrations of such media in the form of waves. Therefore, waves were considered vibrations of a medium made out of several point particles, and the local interactions between two neighboring particles would allow for a perturbation in one point of the medium to be propagated to another point of the medium. More importantly, such effect depends not only on the position of the particle, but also possibly of all other particles or elementary events that make up the medium, and also on all interactions or boundary conditions that such particles need to satisfy. In other words, waves interact non-locally.

Thus, a media and the wave give an example of total (global) dependence in contrast with the particle. The paradox is that an element (a particle) has a property (global dependence) of the whole media. This is impossible in the classical logic. The global dependence (non-local interaction of the whole system) is a property of the structure of the media. An element cannot have such a property of the whole system because an element has no structure. To explain why the paradox is only apparent we start from Kolmogorov's probability measure that is defined at the level of propositional classical logic and set theory.

\section{Probability Space}

Let $\Omega$ be a finite set, $F$ be an algebra over $\Omega$ and $p$ be a real-valued function, $p: F \rightarrow R$. Then $(\Omega, F, p)$ is a probability space, and $p$ a probability measure, if and only if:
K1. $0 \leq p\left(\left\{\omega_{i}\right\}\right) \leq 1, \forall \omega_{i} \in \Omega$
K2. $p(\Omega)=1$
K3. $p\left(\left\{\omega_{i}, \omega_{j}\right\}\right)=p\left(\left\{\omega_{i}\right\}\right)+p\left(\left\{\omega_{j}\right\}\right)$ 
The elements $\omega_{i}$ of $\Omega$ are called elementary probability events or simply elementary events. The elementary events are disjoint sets. Given two sets of elementary probability events $A$ and $B$ the intersection of the two events is given by the expression

$$
p(A \cap B)=p(A, B)=P(A) P(B \mid A)
$$

When the two sets of events are independent we have

$$
p(A \cap B)=p(A, B)=P(A) P(B)
$$

with a trivial density of copula, $c(A, B)=1$.

$$
p\left(e_{1} \cup e_{2}\right)=p(A \text { or } B)=p\left(e_{1}\right)+p\left(e_{2}\right)+p\left(e_{1} \text { and } e_{2}\right)
$$

Now when the events ar dijoint one with the other we have

$$
p\left(e_{1} \cup e_{2}\right)=p(A \text { or } B)=p\left(e_{1}\right)+p\left(e_{2}\right)
$$

\section{The real joint probability for double slit experiment by quantum mechancis is}

$$
p\left(\alpha_{1}, \alpha_{2}\right)=k \cos ^{2}\left(\alpha_{1}-\alpha_{2}\right)
$$

for which copula is

$$
\begin{aligned}
& C\left(F\left(\alpha_{1}\right), F\left(\alpha_{2}\right)\right) \\
& =k \sin ^{2}\left[\frac{1}{2}\left(\frac{\frac{\pi}{2} \pm \sqrt{\left(\frac{\pi}{2}\right)^{2}+\frac{16}{\pi} \arccos \left(\frac{1}{k} \sqrt{F_{1}\left(\alpha_{1}\right)}\right)}}{2}\right)^{2} \frac{\frac{\pi}{2} \mp \sqrt{\left(\frac{\pi}{2}\right)^{2}+\frac{16}{\pi} \arccos \left(\frac{1}{k} \sqrt{F_{2}\left(\alpha_{2}\right)}\right)}}{2}\right. \\
& \left.-\left(\frac{\frac{\pi}{2} \mp \sqrt{\left(\frac{\pi}{2}\right)^{2}+\frac{16}{\pi} \arccos \left(\frac{1}{k} \sqrt{F_{2}\left(\alpha_{2}\right)}\right)}}{2}\right)^{2} \frac{\frac{\pi}{2} \pm \sqrt{\left(\frac{\pi}{2}\right)^{2}+\frac{16}{\pi} \arccos \left(\frac{1}{k} \sqrt{F_{1}\left(\alpha_{1}\right)}\right)}}{2}\right] \\
& =C\left(u_{1}, u_{2}\right)
\end{aligned}
$$

This copula is tabulated as follows:

$$
M 1=\left(\begin{array}{ccccccccc}
1 & 0.846 & 0.7 & 0.561 & 0.43 & 0.307 & 0.192 & 0.088 & 0 \\
0.846 & 1 & 0.969 & 0.899 & 0.805 & 0.692 & 0.56 & 0.401 & 0.125 \\
0.7 & 0.969 & 1 & 0.979 & 0.923 & 0.839 & 0.725 & 0.572 & 0.25 \\
0.561 & 0.899 & 0.979 & 1 & 0.982 & 0.93 & 0.843 & 0.708 & 0.375 \\
0.43 & 0.805 & 0.923 & 0.982 & 1 & 0.982 & 0.927 & 0.82 & 0.5 \\
0.307 & 0.692 & 0.839 & 0.93 & 0.982 & 1 & 0.98 & 0.909 & 0.625 \\
0.192 & 0.56 & 0.725 & 0.843 & 0.927 & 0.98 & 1 & 0.973 & 0.75 \\
0.088 & 0.401 & 0.572 & 0.708 & 0.82 & 0.909 & 0.973 & 1 & 0.875 \\
0 & 0.125 & 0.25 & 0.375 & 0.5 & 0.625 & 0.75 & 0.875 & 1
\end{array}\right)
$$

Now for the dependence element as copula we have that set theory is not sufficient because two disjoint sets can have a probability (evidence) different from the traditional formula.

In a graphic way we see the traditional set theory with dependences by arrows.

Extension of the set theory by evidence theory (Figure 6) in quantum mechanics can be found in the paper of Germano Resconi and others International Journal of Modern Physics C. Vol. 10 No 1 (1999) 29-62. 


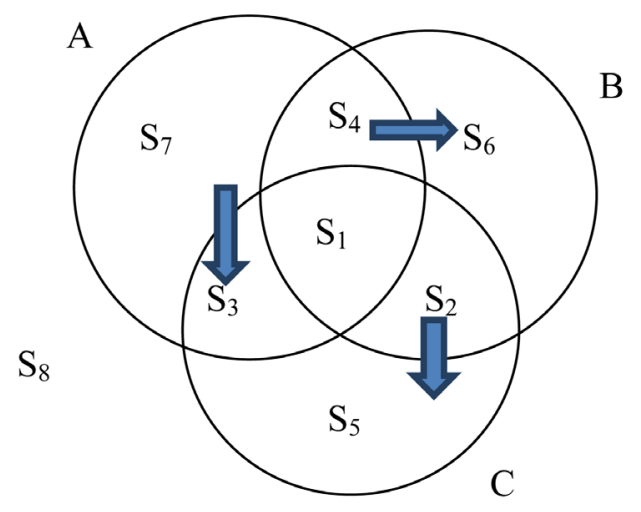

Figure 6. Set theory intersections or elements with dependence.

\section{Conclusions}

Feynman pointed out a logic and mathematical paradox in particle physics [1]. The paradox is that we get for the same entity only local dependence and global dependence at the time.

This contradiction is coming from the dual nature of the particle viewed as a wave. In the first capacity it has only local dependence; in the second (wave) capacity it has a global dependence. The classical logic has difficulties in resolving this paradox. Changing the classical logic to logic makes the paradox apparent. Particle has the local property or zero dependence with other particles, media has total dependence so it is a global unique entity. Now, in set theory, any element is independent from the other so disjoint set has no elements in common. With this condition we have known that the true/false logic can be applied and set theory is the principal foundation. Now with conditional probability and dependence by copula the long distance dependence has an effect on any individual entity that now is not isolate but can have different types of dependence or synchronism (constrain) whose effect is to change the probability of any particle. So particle with different degree of dependence can be represented by a new type of set as fuzzy set in which the boundary is not completely defined or where we cannot separate a set in its parts as in the evidence theory. In conclusion the Feynman paradox and Bell violation can be explained at a new level of complexity by many valued logics and new types of set theory.

\section{References}

[1] Feynman, R.P. (1988). QED: The Strange Theory of Light and Matter. Princeton University Press, Princeton.

[2] Acacio de Barros, J., Garry Oas, G. and Patrik Supper, P. (2014) Negative Probabilities and Counterfactual Reasoning on the Double-Slit Experiment. http://arxiv.org/abs/1412.4888

[3] Feynman, R., Leighton, R. and Sands, M. (2011) The Feynman Lectures on Physics: Mainly Mechanics, Radiation, and Heat, Volume 3. Basic Books, New York. 